Шаповалов В.А. «Высокогорный геофизический институт», г. Нальчик, Россия, vet555_83@mail.ru

\title{
ЧИСЛЕННОЕ МОДЕЛИРОВАНИЕ ФОРМИРОВАНИЯ ГРАДИН В ОБЛАКАХ ПРИ ЕСТЕСТВЕННОМ РАЗВИТИИ И АКТИВНОМ ВОЗДЕЙСТВИИ КРИСТАЛЛИЗУЮЩИМ РЕАГЕНТОМ
}

Введение:

Материалы и методы

исследований:

Все еще остаются малоизученными многие важные аспекты микрофизических процессов в градовых облаках из-за сложности теоретического анализа и экспериментального исследования. Недостаточно изучено взаимодействие термодинамических и микрофизических процессов в мощных конвективных облаках, электрические процессы, взаимодействие реагента с облачной средой. Актуальность исследования обусловлена необходимостью разработки научно обоснованных методов активного воздействия на градовые облака.

В работе применялась трехмерная модель мощных конвективных облаков с детальным описанием термодинамических, микрофизических и электрических процессов. Решение уравнений модели выполнялось конечно-разностным методом по неявной схеме второго порядка точности по пространственным координатам и времени. При задании полей термодинамических параметров атмосферы в начальный момент времени использовались данные аэрологического зондирования или трехмерные данные из глобальной прогностической модели GFS. Результаты исследований и их обсуждение: Результаты численных экспериментов по исследованию взаимодействия физических процессов в мощных конвективных облаках показывают, что важным следствием деформации полей термодинамических характеристик облака под влиянием восходящих потоков, является увеличение времени нахождения градин (или их пути) в области, в которой термодинамические и микрофизические условия благоприятны для их роста. Проведено исследование изменения микроструктурных характеристик облака от концентрации искусственных кристаллов и от места их внесения при засеве градового облака кристаллизующим реагентом. Получено, что в области воздействия отмечается уменьшение радиолокационной отражаемости за счет появления множества кристаллов, из-за вызванной активным воздействием искусственной кристаллизации переохлажденных капель.

Выводы:

Численные эксперименты показали важную роль взаимодействия процессов различных типов в формировании микроструктуры облаков, в частности, в образовании и росте градовых частиц. Наиболее эффективным с точки зрения применения кристаллизующего реагента для уменьшения опасности облака является внесение его в ограниченную область, найденную в результате численных экспериментов. Определено влияние кристаллизующего реагента на микроструктурные параметры облака в результате засева.

Ключевые слова: трехмерная модель, конвективное облако, численные эксперименты, рост градин, кристаллизующий реагент, радиолокационная отражаемость 
Shapovalov V.A.

\section{NUMERICAL SIMULATION OF THE FORMATION OF HAILSTONES IN THE CLOUDS AT THE NATURAL DEVELOPMENT AND AT THE ACTIVE INFLUENCE WITH THE HELP OF CRYSTALLIZING REAGENT}

Introduction:

Many important aspects of microphysical processes in hail clouds are still poorly understood due to the complexity of theoretical analysis and experimental research. The interaction of thermodynamic and microphysical processes in powerful convective clouds, electrical processes, interaction of the reagent with the cloud environment is not sufficiently studied. The relevance of the study is due to the need to develop scientifically valid methods of active impact to hail clouds.

Materials and methods of research:

Results of the research and their discussion:

Conclusions:

Numerical experiments have shown the important role of interaction of processes of various types in the formation of the microstructure of clouds, in particular, in the formation and growth of hail particles. The most effective from the point of view of using the crystallizing reagent to reduce the danger of the cloud is to introduce it into a limited area, found as a result of numerical experiments. The effect of the crystallizing reagent on the microstructural parameters of the cloud as a result of seeding is determined.

Key words: $\quad$ three-dimensional model, convective cloud, numerical experiments, growth of Hailstones, crystallizing reagent, radar reflectivity 


\section{Введение}

Одной из наиболее сложных в физике облаков является градовая проблема. Этой проблеме посвящено множество теоретических и экспериментальных исследований у нас в стране и за рубежом, в ходе которых установлены закономерности образования и развития градовых облаков различных типов, изучено их строение, разработаны методы воздействия на них [1-6]. Тем не менее, до сих пор многие вопросы, связанные с образованием и ростом градин, остаются нерешенными. В частности, все еще остаются малоизученными многие важные аспекты микрофизических процессов в градовых облаках из-за сложности теоретического анализа и экспериментального исследования. Недостаточно изучено взаимодействие термодинамических и микрофизических процессов в мощных конвективных облаках, электрические процессы, взаимодействие реагента с облачной средой. В то же время проблемы усовершенствования существующих и разработки научно-обоснованных методов активного воздействия на градовые облака требуют глубокого изучения этих вопросов.

Представляет научный и практический интерес численное исследование эффективности воздействия кристаллизующим реагентом на градовые облака. Эта проблема рассматривалась во многих работах [7-15]. Недостатком многих исследований является использование моделей с параметризованной микрофизикой, в которых сложно корректно формализовать активное воздействие. В частности, невозможно корректно описать рост искусственных кристалликов и их взаимодействие с облачной средой из-за ограниченности числа классов размеров частиц (от 5 до 15), а значит и их физических свойств (скорость падения, коэффициент коагуляции и т.д.).

В данной работе представлены результаты численных экспериментов по моделированию засева градового облака кристаллизующим реагентом, которые проведены на основе трехмерной модели с детальным учетом термодинамических, микрофизических и электрических процессов.

\section{Материалы и методы исследований}

Для выполнения численных экспериментов использовалась трехмерная модель конвективных облаков с детальным учетом термодинамических, микрофизических и электрических процессов, описание которой подробно изложено в работах [7-10].

Функция $u(x, y, z, m, t)$ в системе уравнений модели облака описывает источник искусственных кристаллов, а ее вид зависит от того, какими средствами осуществляется воздействие. В данной работе моделировался монодисперсный источник:

$$
u(x, y, z, m, t)=\gamma(t) \cdot \delta\left(m-m_{u c m}\right) \cdot \delta\left(x-x_{u c m}\right) \cdot \delta\left(y-y_{u c m}\right) \cdot \delta\left(z-z_{u c m}\right)
$$


где $\gamma(t)-\quad$ функция, описывающая интенсивность источника искусственных кристаллов;

$m_{u c m}-\quad$ масса искусственного кристалла;

$x_{u c m}, y_{u c m}, z_{u c m}-$ координаты источника в облаке;

$\delta$ _ $\quad$ дельта-функция. Моделировались также многоточечные источники, представляющие линейный или площадной засев.

Контрольным значением в численных экспериментах служило число крупных градовых частиц, находящихся в облаке за время развития:

$$
F[u]=\int_{0}^{L_{x}} \int_{0}^{L_{y}} \int_{0}^{L_{z}} \int_{0}^{\infty} \int_{m_{k}}^{T} f_{2}(x, y, z, m, t) d x d y d z d m d t
$$

где $m_{k}-$

масса градины, способной достичь поверхности земли и нанести ущерб [8].

Для выяснения влияния активного воздействия на градоопасность облака было просчитано множество вариантов, в которых варьировались параметры источника искусственных ледяных кристаллов. Исследовалось изменение микроструктурных характеристик облака от концентрации искусственных кристаллов, от места воздействия, времени их внесения.

В каждой серии численных экспериментов предварительно проводился расчет формирования микроструктуры облака при естественном развитии (функция $u(x, y, z, m, t)=0)$. Было получено значение числа крупных градовых частиц, соответствующее естественному развитию облака. Затем проводились расчеты по перебору пространственного положения источника и его мощности.

Моделирование эволюции ансамбля облачных частиц с помощью разработанной модели проводилось в трехмерной прямоугольной области, выделенной в тропосфере. В области рассчитываются поля термодинамических параметров, таких как: давление, температура, влажность, а также характеристики воздушных потоков и другие параметры облака.

При задании полей термодинамических параметров атмосферы в начальный момент времени использовались как одномерные (горизонтально однородные) данные аэрологического зондирования, так и трехмерные данные из GFS модели [10].

Моделировался случай активного воздействия, в котором внесение реагента осуществляется на уровне $-10{ }^{\circ} \mathrm{C}(\mathrm{z}=5$ км) во фронтальной части облака. С использованием данных о содержании $\mathrm{AgI}$ в современных противоградовых ракетах, была определена начальная концентрация искусственных частиц в шлейфе за ракетой. 
Решение уравнений модели выполняется численными методами и требует аппроксимации задачи на пространственной сетке. Вследствие этого минимальная пространственная сеточная ячейка модели ограничена шагами по высоте и горизонтали. В силу дискретного представления задачи начальная концентрация в шлейфе за ракетой должна быть пересчитана на объем, соответствующий минимальной сеточной ячейке. Время расширения шлейфа до объема ячейки модели оценивалось при расчетах на более мелких сетках с шагами: 0,$1 ; 1$; и 10 м. Источник в начальный момент времени из-за разбиения расчетной области на ячейки имеет объем $\mathrm{V}_{\text {ист }}=\mathrm{dx} * \mathrm{dy} * \mathrm{dz}\left(\mathrm{M}^{3}\right)$.

Трансформация облачного объема, содержащего искусственные кристаллы, прослеживалась в последовательные моменты времени 1, 3, 5, 10 минут и более.

\section{Результаты исследований и их обсуждение}

Максимальные значения скорости восходящих потоков на стадии появления первого радиоэхо (-10 dBZ) достигают 20 м/с на уровне $4,5 \div 5$ км. Картина воздушных потоков представлена на рисунке 1 изолиниями вертикальных скоростей $W(x, y, z)$. При анализе полей термодинамических характеристик облака отмечалась их деформация под влиянием вертикальных потоков воздуха. Поле температуры, как и поле скоростей потоков воздуха изменяется со временем в облаке.

В серии расчетов активное воздействие на градовое облако моделировалось включением точечного источника искусственных кристаллов с постоянной интенсивностью равной $106 \mathrm{M}^{-3} \mathrm{c}^{-1}$ частиц, действующего в течение 1 минуты, начиная с момента времени $\mathrm{t}=24$ мин. Искусственные частицы, выросшие в результате активации частиц реагента, считаются примерно одинаковыми, и относятся к первому классу сетки размеров, примененной в модели. Размер искусственных частиц составлял 2 мкм. По результатам расчетов определено, что воздействие более эффективно в области повышенной водности на температурном уровне $-10{ }^{\circ} \mathrm{C}$.

На рисунке 2 приведены в двух ракурсах результаты другого случая моделирования активного воздействия на градовое облако льдообразующим реагентом (воздействие моделировалось на 25 минуте развития).

В области воздействия в представленных случаях отмечается уменьшение радиолокационной отражаемости за счет появления множества кристаллов, из-за вызванной воздействием искусственной кристаллизации переохлажденных капель. На рисунках $2(a, \sigma)$ приведено одно и то же облако с разных ракурсов при естественном развитии (вид сбоку и вид сверху под небольшим углом). На рисунках 2 (в,г) приведено это же облако, но уже при воздействии на него кристаллизующим реагентом. Видно, что в области воздействия радиолокационная отражаемость уменьшается, образуется «провал». 


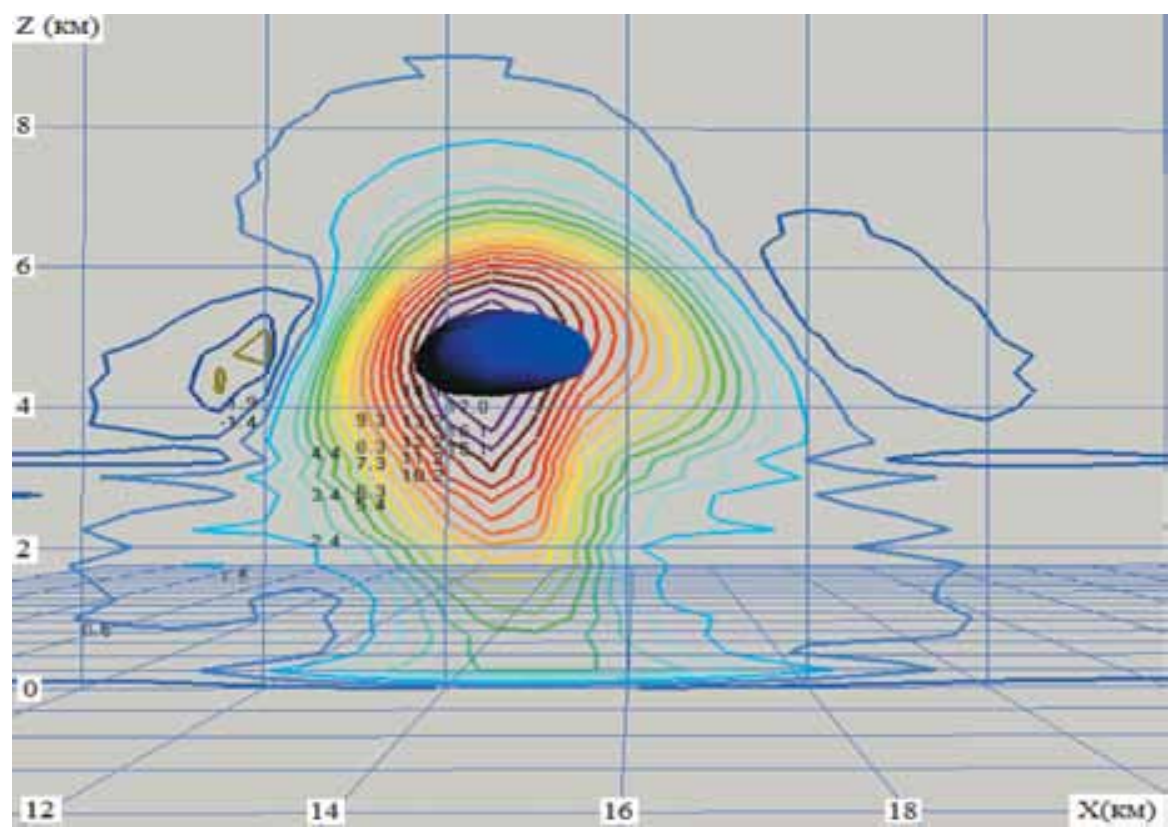

Puc. 1.

Вертикальный разрез мощного конвективного облака. Изоповерхность водности (7 r/m $\left.\mathbf{m}^{3}\right)$ и изолинии вертикальных потоков воздуха (W) на 30-й минуте развития.

Fig. 1. A vertical cross-section of the powerful convective cloud. Isosurface liquid water content $\left(7 \mathrm{~g} / \mathrm{m}^{3}\right)$ and isoline of vertical air flows $(\mathrm{W})$ at the 30th minute of evolution.

Остановимся на некоторых результатах исследований формирования микроструктуры градовых облаков, а также движения и роста градовых частиц в них, полученных на основе моделей. Как показали результаты расчетов, взаимодействие процессов различных типов в этих облаках играет важную роль в формировании микроструктуры облаков, в частности, в образовании и росте градовых частиц [7]. Вследствие деформации полей термодинамических параметров под влиянием динамических процессов в мощных конвективных облаках формируется зона, в которой происходит образование и 


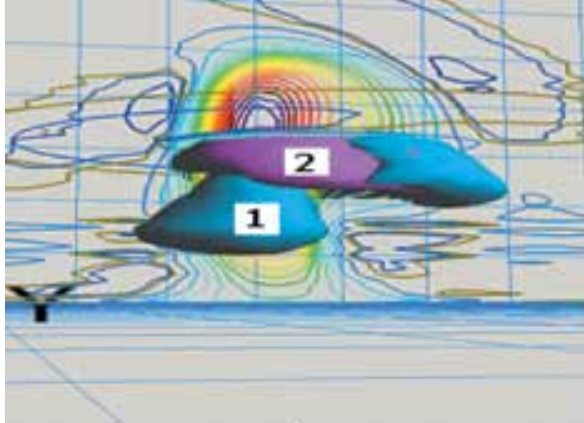

a)

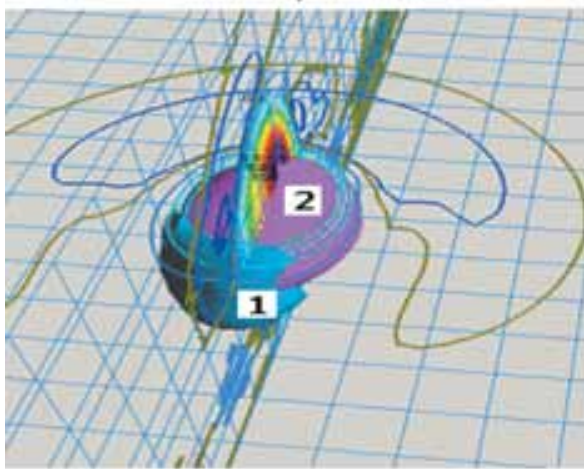

6)

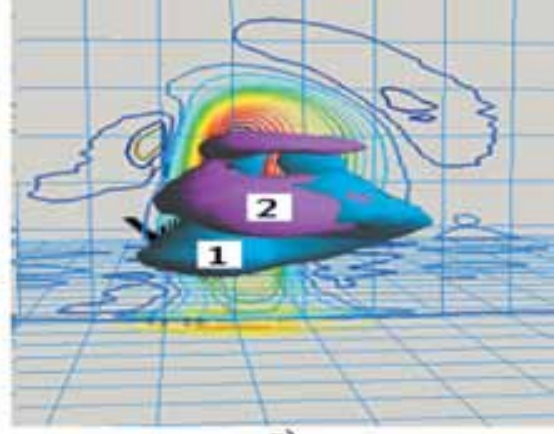

B)

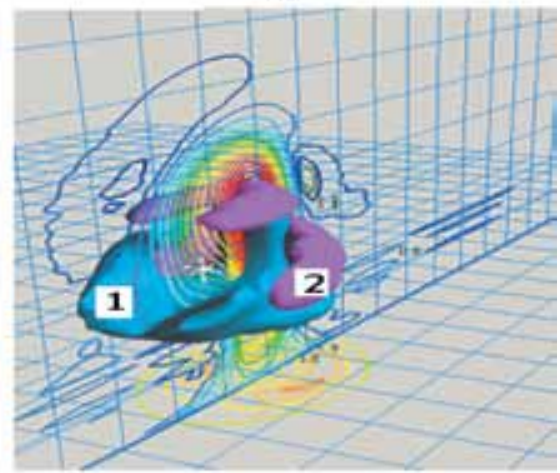

r)

Pис. 2.

Облако, виды с двух ракурсов на 30-й минуте после воздействия. Показаны изоповерхности области капель (1), крупных ледяных кристаллов (2) и изолинии восходящих и нисходящих потоков. Варианты а), б) соответствуют естественному развитию облака, варианты в), г) - облаку после воздействия.

Fig. 2. The cloud, views from two angles at the 30th minute after exposure. The isosurfaces of the droplet region (1), large ice crystals (2), and isolines of ascending and descending flows are shown. Options a), b) correspond to the natural evolution of the cloud, options c), d) - the cloud after active impact.

рост градовых частиц. Эта зона располагается между изотермами $-10 . \div-30{ }^{\circ} \mathrm{C}$ и в области интенсивных восходящих потоков. С применением разработанной трехмерной модели в данной работе получено, что образование первичных градовых частиц происходит в верхней части этой зоны. В дальнейшем под влиянием горизонтальной составляющей вектора скорости воздушных потоков и силы тяжести происходит смещение градин от места образования и вниз. При этом увеличиваются размеры градин за счет захвата поступающих снизу в эту зону переохлажденных капель, также происходит увеличение их 
концентрации за счет образования новых градин в нижних частях этой зоны. Важно отметить, что следствием деформации полей термодинамических характеристик облака под влиянием восходящих потоков, является увеличение времени нахождения градин (или их пути) в области, в которой термодинамические и микрофизические условия благоприятны для их роста.

В работе исследованы физические процессы, приводящие к достижению эффекта при воздействии. В частности, искусственные кристаллы активизируют процесс замерзания переохлажденной капельной фракции, за счет которой растут градины. Появление большого количества мелких кристаллов приводит к нарушению равновесного состояния между жидкокапельной и кристаллической фазами, начинается сублимационный рост кристаллов за счет перегонки пара с капель. В свою очередь, процесс испарения капель приводит к интенсификации их кристаллизации, кроме этого в результате увеличения размеров кристаллов происходит интенсивный захват ими переохлажденных капель.

Таким образом, внесение частиц льдообразующего реагента по предложенному способу приводит к замораживанию воды в зоне роста градовых частиц, т.е. к ухудшению условий роста градовых частиц. При этом не требуется чрезмерных затрат реагента, так как «работает» неустойчивость системы переохлажденные капли - кристаллы. Наиболее эффективным с точки зрения использования реагента и уменьшения градоопасности облака при проведении противоградовых работ является их внесение в ограниченную область, наиболее чувствительную к воздействию.

Очевидно, что эффективное решение проблемы активных воздействий на градовые облака невозможно без глубоких исследований закономерностей формирования макро- и микроструктурных характеристик облаков на основе полных физико-математических моделей, учитывающих взаимодействие процессов различных видов [16-19].

Рассмотрим результаты расчетов моделирования переноса и распространения частиц реагента в конвективном облаке, полученные с использованием трехмерной модели. Их пример представлен на рисунке 3, на котором изображен расширившийся вследствие турбулентной диффузии шлейф от противоградовой ракеты через 10 мин после воздействия. Представлены область формирования и роста градин (изоповерхность водности 5 г/ $\mathrm{M}^{3}$ ) обозначена цифрой 1 , и шлейф ракеты (изоповерхность концентрации искусственных частиц $\left.10^{3} 1 / \mathrm{m}^{3}\right)$ - обозначена цифрой 2.

В численных экспериментах варьировались: количество точечных источников и концентрация ледяных кристалликов в них, место и время внесения реагента. Искусственные частицы переносятся воздушными потоками, а расширение первоначально обработанной области происходит под влиянием турбулентной диффузии. Степень расширения засеянного объема определяется величиной коэффициента турбулентной диффузии. 


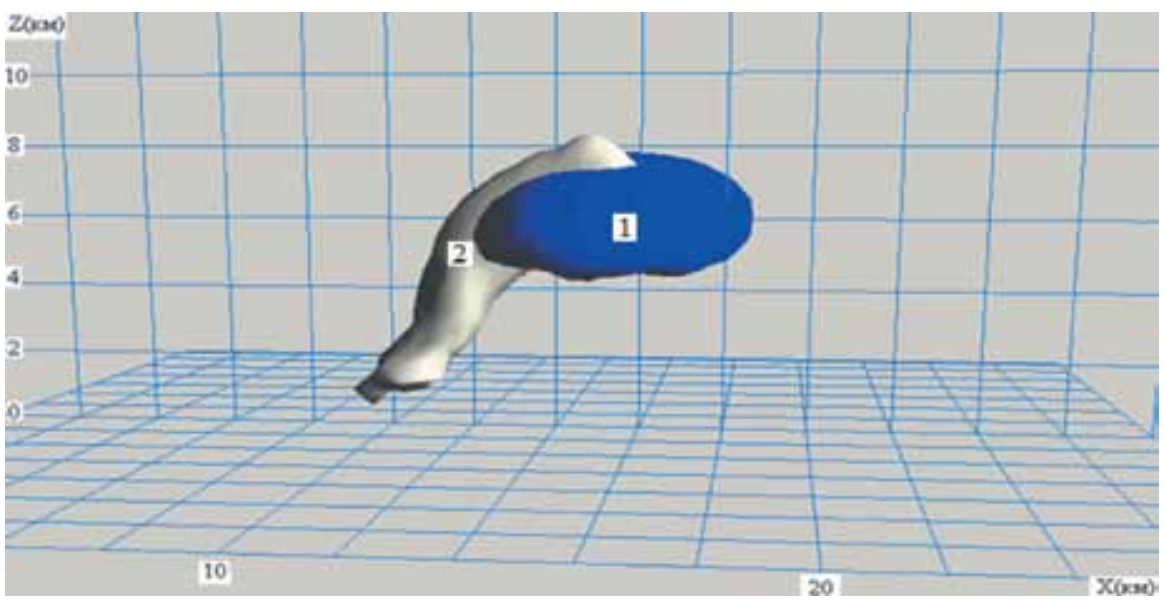

Pис. 3.

Распространение кристаллизующего реагента при ракетном засеве через $\Delta T=10$ мин. Среднее значение $К$ в центре облака составляет $500 \mathrm{~m}^{2} / \mathrm{c}$.

Fig. 3. The distribution of crystallizing reagent during rocket seeding after $\Delta T=10 \mathrm{~min}$. Average value of $K$ in the center of the cloud is $500 \mathrm{~m}^{2} / \mathrm{s}$.

Полученные данные показывают, что распространение искусственных частиц при значениях коэффициента турбулентной диффузии, которые наблюдаются на периферии облака, вне центральной части, идет медленнее. При этом в течение времени 2-5 минут реагент остается в ограниченном объеме по траектории полета противоградовой ракеты.

\section{Выводы}

На основе численных экспериментов на основе трехмерной модели градового облака получены следующие результаты:

Определено влияние кристаллизующего реагента на микроструктурные параметры облака в результате засева. Определена область, воздействие в которую приводит к существенному эффекту по предотвращению образования крупных градин. Область внесения реагента по результатам расчетов должна быть расположена в центре облака, на температурном уровне $-10 . \div .-12^{\circ} \mathrm{C}$, в области умеренных восходящих потоков $\left(5^{-10} \mathrm{M} / \mathrm{c}\right)$ и больших значений коэффициента турбулентной диффузии $\left(>300 \mathrm{~m}^{2} \mathrm{c}^{-1}\right)$.

Физика влияния искусственных ледяных частиц на микроструктуру облака заключается в том, что воздействие в наиболее водной части облака мелкими искусственными кристалликами в концентрации $106 \mathrm{~m}^{-3}$ приводит к частичной или полной ликвидации там жидкокапельной фракции, в результате чего число крупных градин, образующихся в облаке, уменьшается. 
Исследовано распространение искусственных ледяных кристаллов в облаке с учетом фактических потоков, термодинамических условий, взаимодействия с облачной средой. В течение времени до 5 минут реагент остается в ограниченном объеме по траектории засева.

\section{Библиографический список}

1. Сулаквелидзе, Г. К. Ливневые осадки и град. Л.: Гидрометеоиздат. $1967.412 \mathrm{c}$.

2. Хоргуани В.Г. Микрофизика зарождения и роста града. М.: Гидрометеоиздат, 1984. $184 \mathrm{c}$.

3. Browning, K.A., Foote G.B. Airflow and hail growth in super cell storms and some implications for hail suppression. Quart. J. Roy. Met. Soc. № 102. 1975. P. 499-534.

4. Pruppacher H.R., Klett J.D. Microphysics of clouds and precipitation// D.Reidel Pub. Co., 1978. 714 p.

5. Simpson, J. The National Hail Research Experiment Report on the Alberta Hail Project. NCAR Technical Note. 1976. doi:10.5065/ D64Q7RX8.

6. Zakinyan R. G. On the theory of hailstone growth. Izv. Atmos. Ocean. Phys. V. 44 I. 2. 2008. P 207-212. https://doi.org/10.1134/ S0001433808020084.

7. Ашабоков Б.А., Федченко Л.М., Шаповалов А.В., Шаповалов В. А. Физика облаков и активных воздействий на них. - Нальчик: «Печатный двор», 2017. 240 c.

8. Ashabokov, B.A., Shapovalov A.V. A numerical model for controlling the formation of the microstructure of a hail cloud. Izvestiya Atmospheric and Ocean Physics V. 32, I. 3, 1996, P. 333-338.

9. Ashabokov, B.A., Shapovalov, A.V., Kuliev, D.D., Prodan, K.A., Shapovalov, V.A. Numerical simulation of thermodynamic, microstructural, and electric characteristics of convective clouds at the growth and mature stages. Radiophysics and Quantum Electronics. Volume 56, Issue 11. 2014. P. 811-817

10. Ашабоков Б.А., и др. Физика градовых облаков и активных воздействий на них: состояние и направления развития. Нальчик: Печатный двор, 2013. 216 с.

11. Веремей, Н. Е., Довгалюк Ю. А., Затевахин М. А., Игнатьев А. А., Морозов В. Н., Пастушков Р. С. Описание базовой численной нестационарной трехмерной модели конвективного облака // Труды ГГО, Вып. 582. 2016. С. 45-91.

12. Владимиров, С. А., Пастушков Р. С. Комплексный метод активных воздействий на конвективные облака с целью регулирования осадков. Трёхмерное численное моделирование // Труды ГГО. Вып. 582. 2016. С. 116-127.

13. Довгалюк, Ю. А., Веремей Н. Е., и др. Концепция разработки численной нестационарной трехмерной модели эволюции осадкообразующего конвективного облака в естественных ус- 
ловиях и при активных воздействиях// Труды ГГО, Вып. 282. 2016. C. 7-44.

14. Danielsen, E.F., Bleck R. and Morris B.A. Hail growth by stochastic collection in a Cumulus model//J. Atmos. Sci. Vol.29. № 1. 1972. P. 135-155.

15. Farley, R.D., Orville H.D. Numerical modeling of hailstorms and hailstone growth. Part I: Preliminary model verification and sensitivity tests. J. Climate Appl. Meteor., 25, 1986. P. 2014-2035.

16. Khain, A.P., Lynn B.H. Simulation of a supercell storm in clean and dirty atmosphere using weather research and forecast model with spectral bin microphysics, J. Geophys. Res., 114. 2009. P. 29632982.

17. Meyers, M. P., Walko R. L., Harrington J. Y., Cotton W. R. New RAMS cloud microphysics parameterization. Part II: The two-moment scheme // Atmos. Research. V. 45. № 1. 1997. P. 3-29.

18. Miller, K.; Gadian A.; Saunders C.; Latham J.; Christian H. Modelling and observations of thundercloud electrification and lightning, ATMOS RES, 58, 2001. P. 89-115.

19. Orville, H.D., Kopp F.J. Numerical simulation of the life history of a hailstorm. J. Atm. Sci. Vol.34. № 10. 1977. P. 1596-1618.

\section{References}

1. Sulakvelidze, G. K. Livnevye osadki i grad (Rainfall and hail). L.: Gidrometeoizdat. 1967. 412 p. (in Russ)

2. Horguani V.G. Mikrofizika zarozhdenija i rosta grada (Microphysics of nucleation and hail growth). Moscow: Gidrometeoizdat, 1984. 184 p. (in Russ)

3. Browning, K.A., Foote G.B. Airflow and hail growth in super cell storms and some implications for hail suppression. Quart. J. Roy. Met. Soc. 1975. № 102. P. 499-534.

4. Pruppacher H.R., Klett J.D. Microphysics of clouds and precipitation// D.Reidel Pub. Co., 1978. 714 p.

5. Simpson, J., The National Hail Research Experiment Report on the Alberta Hail Project. NCAR Technical Note , 1976. doi:10.5065/ D64Q7RX8.

6. Zakinyan R. G. On the theory of hailstone growth. Izv. Atmos. Ocean. Phys. V. 44 I. 2. 2008. P 207-212. https://doi.org/10.1134/ S0001433808020084.

7. Ashabokov, B.A., Fedchenko L.M., Shapovalov A.V., Shapovalov V. A. Fizika oblakov i aktivnyh vozdejstvij na nih (Physics of clouds and active influences on them). - Nal'chik: «Pechatnyj dvor», 2017. 240 p. (in Russ)

8. Ashabokov, B.A., Shapovalov A.V. A numerical model for controlling the formation of the microstructure of a hail cloud. Izvestiya - Atmospheric and Ocean Physics V. 32, I. 3, 1996, P. 333-338.

9. Ashabokov, B.A., Shapovalov, A.V., Kuliev, D.D., Prodan, K.A., Shapovalov, V.A. Numerical simulation of thermodynamic, microstructural, and electric characteristics of convective clouds at the 
growth and mature stages. Radiophysics and Quantum Electronics. Volume 56, Issue 11. 2014. P. 811-817

10. Ashabokov B.A. i dr. Fizika gradovyh oblakov i aktivnyh vozdejstvij na nih: sostojanie i napravlenija razvitija (The physics of hail clouds and active influences on them: the state and directions of development). Nal'chik: Pechatnyj dvor, 2013. 216 p. (in Russ).

11. Veremej, N. E., Dovgaljuk Ju. A., Zatevahin M. A., Ignat'ev A. A., Morozov V. N., Pastushkov R. S. Opisanie bazovoj chislennoj nestacionarnoj trehmernoj modeli konvektivnogo oblaka (Description of the basic numerical nonstationary three-dimensional model of a convective cloud) // Trudy GGO, V. 582. 2016. P. 45-91.

12. Vladimirov, S. A., Pastushkov R. S. Kompleksnyj metod aktivnyh vozdejstvij na konvektivnye oblaka s cel'ju regulirovanija osadkov. Trjohmernoe chislennoe modelirovanie (Complex method of active influences on convective clouds for the purpose of precipitation regulation. Three-dimensional numerical modeling) // Trudy GGO. V. 582. 2016. P. 116-127. (in Russ).

13. Dovgaljuk, Ju. A., Veremej N. E., i dr. Koncepcija razrabotki chislennoj nestacionarnoj trehmernoj modeli jevoljucii osadkoobrazujushhego konvektivnogo oblaka v estestvennyh uslovijah i pri aktivnyh vozdejstvijah (The concept of development of a numerical non-stationary three-dimensional model for the evolution of the sedimentary convective cloud under natural conditions and under active influences) // Trudy GGO, V. 282. 2016. P. 7-44. (in Russ).

14. Danielsen, E.F., Bleck R. and Morris B.A. Hail growth by stochastic collection in a Cumulus model // J. Atmos. Sci. Vol.29. № 1. 1972. P. 135-155.

15. Farley, R.D., Orville H.D. Numerical modeling of hailstorms and hailstone growth. Part I: Preliminary model verification and sensitivity tests. J. Climate Appl. Meteor., 25, 1986. P. 2014-2035.

16. Khain, A.P., Lynn B.H. Simulation of a supercell storm in clean and dirty atmosphere using weather research and forecast model with spectral bin microphysics, J. Geophys. Res., 114. 2009. P. 29632982.

17. Meyers, M. P., Walko R. L., Harrington J. Y., Cotton W. R. New RAMS cloud microphysics parameterization. Part II: The two-moment scheme // Atmos. Research. V. 45. № 1. 1997. P. 3-29.

18. Miller, K., Gadian A., Saunders C., Latham J., Christian H. Modelling and observations of thundercloud electrification and lightning, Atmos. Res., 58, 2001. P. 89-115.

19. Orville, H.D., Kopp F.J. Numerical simulation of the life history of a hailstorm. J. Atm. Sci. Vol.34. № 10. 1977. P. 1596-1618.

Рукопись поступила в редакцию: 14.05 .2018 , принята к публикации: 26.08.2018 


\section{6 авторе}

Шаповалов Виталий Александрович, кандидат физико-математических наук, старший научный сотрудник, отдел фризики облаков, ФГБУ "Высокогорный геофизический институт", Телефон: +79187855350, ORCID: https://orcid.org/0000-0002-9701-6820 Scopus ID: 57190 966150, Researcher ID: J-9696-2015, E-mail: atajuk@mail.ru.

\section{About the author}

Shapovalov Vitaly Alexandrovich, candidate of physico-mathematical Sciences, senior researcher, Department of cloud physics, Federal state budget institution "High-Mountain geophysical Institute", Phone: 89187855350, ORCID: https://orcid.org/0000-0002-9701-6820 Scopus ID: 57190966150, Researcher ID: J-9696-2015, E-mail: atajuk@mail.ru. 